\title{
miR-194-5p serves a suppressive role in human keloid fibroblasts via targeting NR2F2
}

\author{
QIJUN XU ${ }^{1}$ and SHENGJUN JIANG ${ }^{2}$ \\ ${ }^{1}$ Department of Plastic Surgery, The Third Hospital of Wuhan; ${ }^{2}$ Department of Stomatology, \\ Renmin Hospital of Wuhan University, Wuhan, Hubei 430060, P.R. China
}

Received February 4, 2020; Accepted September 11, 2020

DOI: $10.3892 / \mathrm{mmr} .2020 .11695$

\begin{abstract}
Keloids are a skin fibrotic disease that cause a number of problems for reconstructive surgeons. MicroRNAs (miRs) are crucial for the development of keloids. The present study aimed to investigate the function of the miR-194-5p/nuclear receptor subfamily 2 group $F$ member 2 (NR2F2) interactome in human keloid fibroblasts. Microarray analysis was performed to identify key genes that may participate in keloid progression. The expression levels of miR-194-5p and NR2F2 mRNA in normal human skin fibroblasts (HSFs) and human keloid fibroblasts (KEL-FIBs) were measured via reverse transcription-quantitative PCR. Furthermore, cell proliferation, apoptosis, migration and invasion were assessed in KEL-FIB cells. Following NR2F2 knockdown and miR-194-5p inhibition, NR2F2 expression was measured via western blotting. The microarray analysis identified NR2F2 as a key gene related to keloids. The regulatory association between miR-194-5p and NR2F2 was identified using TargetScan Human (version 7.2) and verified by performing a dual-luciferase reporter assay. miR-194-5p expression was decreased in KEL-FIB cells compared with HSF cells, and miR-194-5p overexpression inhibited the aggressive phenotypes of KEL-FIB cells compared with the negative control group. Meanwhile, NR2F2 expression was negatively correlated with miR-194-5p expression. NR2F2 knockdown and miR-194-5p overexpression displayed similar effects on KEL-FIB cells. Moreover, NR2F2 knockdown effectively reversed miR-194-5p inhibitor-mediated effects in keloid fibroblasts. The present study indicated that the novel miR/194-5p/NR2F2 interactome might be involved in the progression of keloid aggression and may serve as a potential therapeutic target for human keloid in the future.
\end{abstract}

Correspondence to: Dr Shengjun Jiang, Department of Stomatology, Renmin Hospital of Wuhan University, 99 Zhangzhidong Road, Wuchang, Wuhan, Hubei 430060, P.R. China

E-mail: 2532118505@qq.com

Key words: keloid, microRNA-194-5p, nuclear receptor subfamily 2 group F member 2

\section{Introduction}

Keloids, characterized by excessive proliferation of fibroblasts, are one of the most common and refractory diseases in dermatology (1). Keloids are abnormal tissue scars that form when fibroblasts over accumulate, resulting in a large amount of collagen fibrin and extracellular matrix deposition (2). Patients with keloids frequently present with pain, itching and occasionally loss of shin function, which affect the physical and psychological quality of life of patients (3-5). Despite the development of clinical therapies, such as interferon, radiation, surgical resection and hormones, the treatment of keloids is still limited due to insufficient research on the underlying pathogenic mechanism $(3,6,7)$. Therefore, exploring the key genes and microRNAs (miRNAs/miRs) associated with keloid pathogenesis might be useful for the development of novel therapeutic targets and for the theoretical guidance of keloids.

miRNAs/miRs are a group of essential regulatory factors that negatively regulate the expression of their target genes to modulate a number of biological processes (8-12). Increasing evidence has suggested that abnormally expressed miRNAs are associated with diverse cellular processes, including cell apoptosis, differentiation, proliferation and extracellular matrix production (13-16). A previous study demonstrated that miRNA profiles in keloid fibroblasts were different compared with those in normal fibroblasts via microarray analysis (17). Liu et al (18) also demonstrated that the expression of miRNAs was altered in keloid tissues, which might contribute to the development of keloids via regulating signaling pathways associated with scar wound healing.

Nuclear receptor subfamily 2 group F member 2 (NR2F2) is a member of the steroid/thyroid nuclear hormone receptor superfamily (18). Aberrant activation of NR2F2 is related to the occurrence of cancer, including lung, breast and prostate cancer (19-21). NR2F2 overexpression augmented angiogenesis, tumor growth and malignant progression by restraining the TGF- $\beta$-induced growth barrier in prostate cancer (22). However, the effect of NR2F2 on the development of keloids, benign skin tumors, is not completely understood.

The present study aimed to investigate the roles of miR-194-5p and NR2F2 in keloids using bioinformatics analysis and cell functional experiments. Our findings may be useful to provide a novel therapeutic target for keloids. 


\section{Materials and methods}

Microarray analysis. The GSE7890 dataset (23) from the Gene Expression Omnibus database (www.ncbi.nlm.nih. gov/gds) demonstrated the gene profiling of keloid fibroblasts. The GSE7890 dataset included five keloid fibroblasts samples and five healthy fibroblasts samples. Differentially expressed genes (DEGs) with an adjusted P-value $<0.05$ were selected out and uploaded to Search Tool for the Retrieval of Interacting Genes/Proteins (STRING; string-db.org) and Metascape (metascape.org/gp/index.html\#/main/step1) to perform Gene Ontology (GO) and pathway enrichment analyses.

Cell culture and cell transfection. The human normal skin fibroblast (HSF) cell line was purchased from BeNa Culture Collection (cat. no. 338008) and the human keloid fibroblast (KEL-FIB) cell line was purchased from American Type Culture Collection (cat. no. CRL-1762). Cells were maintained in DMEM (Gibco; Thermo Fisher Scientific,Inc.) supplemented with $10 \%$ FBS (Gibco; Thermo Fisher Scientific, Inc.) at $37^{\circ} \mathrm{C}$ with 5\% $\mathrm{CO}_{2}$. miR-194-5p mimic, miR-194-5p inhibitor, small interfering (si)RNA targeting NR2F2 (si-NR2F2), miR-mimic negative control (NC) and miR-inhibitor NC plasmids were purchased from Guangzhou RiboBio Co., Ltd. At 30\% confluence, KEL-FIB cells were transfected with miR-194-5p mimic $(100 \mathrm{nM})$, miR-194-5p inhibitor (100 nM), si-NR2F2 (50 nM) or $50 \mathrm{nM}$ miR-mimic and $50 \mathrm{nM}$ miR-inhibitor NC using Lipofectamine $^{\circledR} 2000$ (Invitrogen; Thermo Fisher Scientific, Inc.) in serum-free DMEM. Following incubation for $4 \mathrm{~h}$ at $37^{\circ} \mathrm{C}$, serum-free medium was replaced with fresh DMEM supplemented with $10 \%$ FBS. At $48 \mathrm{~h}$ post-transfection, transfection efficiency was assessed via reverse transcription-quantitative PCR (RT-qPCR). The sequences of plasmid are presented in Table SI.

$R T-q P C R$. Total RNA was isolated from HSF and KEL-FIB cells using TRIzol ${ }^{\circledR}$ (Invitrogen; Thermo Fisher Scientific, Inc.). Total RNA $(1 \mu \mathrm{g})$ was reverse transcribed into cDNA using the GoScript Reverse Transcription System kit (cat. no. A5001; Promega Corporation) at $70^{\circ} \mathrm{C}$ for $5 \mathrm{~min}, 25^{\circ} \mathrm{C}$ for $5 \mathrm{~min}, 42^{\circ} \mathrm{C}$ for $60 \mathrm{~min}$ and $70^{\circ} \mathrm{C}$ for $15 \mathrm{~min}$. Subsequently, qPCR was performed using a C1000 Thermal Cycler (Bio-Rad Laboratories, Inc.) and SYBR Premix Ex Taq II (cat. no. RR820A; Takara Biotechnology Co., Ltd.). The PCR conditions were specifically shown as: Pre-denaturation at $95^{\circ} \mathrm{C}$ for $30 \mathrm{sec}$, and 40 cycles of denaturation at $95^{\circ} \mathrm{C}$ for $5 \mathrm{sec}$ and annealing at $60^{\circ} \mathrm{C}$ for $30 \mathrm{sec}$. The sequences of primers used in the present study are presented in Table I. miRNA and mRNA expression levels were normalized to the internal reference genes U6 and GAPDH, respectively. The relative expression of miRNA and mRNA was calculated with the $2^{-\Delta \Delta C q}$ method (24).

Cell proliferation assay. Transfected KEL-FIB cells were seeded $\left(3 \times 10^{5}\right.$ cells/well) into a 96-well plate containing $100 \mu 1$ DMEM per well. Following incubation for 24,48 or 72 h, $10 \mu \mathrm{l}$ Cell Counting Kit-8 (CCK-8) reagent (Dojindo Molecular Technologies, Inc.) in $100 \mu 1$ DMEM medium was added to each well for $2 \mathrm{~h}$ at $37^{\circ} \mathrm{C}$. The optical density values
Table I. Sequences of primers used for reverse transcription-quantitative PCR.

\begin{tabular}{ll}
\hline Gene & \multicolumn{1}{c}{ Sequence $\left(5^{\prime} \rightarrow 3^{\prime}\right)$} \\
\hline miR-194-5p & F: ACACTCCAGCTGGGTGTAACAG \\
& AGCAACTCC \\
& R: TGGTGTCGTGGAGTCG \\
NR2F2 & F: GGCAATGGTAGTCAGCACG \\
& R: ACCCATGATGTTGTTGGGCT \\
GAPDH & F: GTCAAGGCTGAGAACGGGAA \\
& R: AAATGAGCCCAGCCTTCTC \\
U6 & F: TGCGGGTGCTCGCTTCGGCAGC \\
& R: CCAGTGCAGGGTCCGAGGT
\end{tabular}

miR, microRNA; NR2F2, nuclear receptor subfamily 2 group $\mathrm{F}$ member 2; F, forward; R, reverse.

were measured at a wavelength of $450 \mathrm{~nm}$ using a microplate reader.

Flow cytometry analysis of cell apoptosis. To assess cell apoptosis, the Annexin V-FITC Apoptosis Staining/Detection kit (cat. no. ab14085; Abcam) was used according to the manufacturer's protocol. Then, cell apoptosis was detected by flow cytometry on a FACSCalibur Flow Cytometer (BD Biosciences), and the apoptosis rate was analyzed by the CellQuest Pro software (version 5.1; Becton-Dickinson). Transfected KEL-FIB cells $\left(1 \times 10^{4}\right)$ were collected and stained with Annexin V-FITC and PI. The rate of apoptosis was calculated as the sum of late apoptosis (Q1-UR) and early apoptosis (Q1-LR).

Wound healing assay. Transfected KEL-FIB cells were plated (1x10 6 cells/well) in 6-well plates. At $90 \%$ confluence (Fig. S1), cells were cultured in serum-free medium. After $12 \mathrm{~h}$, a scratch in the center of the cell monolayer was made using a pipette tip. Cells were washed three times with PBS to remove cell fragments. Subsequently, cells were cultured in serum-free DMEM. Images of the wounds were captured at 0,24 and $48 \mathrm{~h}$ under a light microscope (magnification, x100; Leica Microsystems $\mathrm{GmbH}$ ). The wound width was measured using ImageJ software version 1.46 (National Institutes of Health) to assess cell migration. The rate of cell migration was calculated as the ratio of the migration distance at 24 or $48 \mathrm{~h}$ to the wound width at $0 \mathrm{~h}$.

Transwell invasion assay. Transwell upper chambers were pre-coated with $5 \mathrm{mg} / \mathrm{ml}$ Matrigel at $4^{\circ} \mathrm{C}$ overnight (Corning, Inc.). Subsequently, transfected KEL-FIB cells were seeded $\left(2 \times 10^{5}\right.$ cells/well) in serum-free DMEM into the upper chambers. DMEM supplemented with $10 \%$ FBS was plated in the lower chambers. Following incubation for $48 \mathrm{~h}$, the medium was removed, and invading cells were fixed in ice-cold $100 \%$ methanol for $5 \mathrm{~min}$ at $4^{\circ} \mathrm{C}$, stained with $0.1 \%$ crystal violet for $3 \mathrm{~min}$ at room temperature and washed with PBS. Invading cells on the lower surface of the membrane were observed in 5-7 randomly selected fields of view using a light microscope (Nikon Corporation) at x100 magnification. 
Dual-luciferase reporter assay. The binding site between NR2F2 3'-untranslated region (UTR) and miR-194-5p was predicted using TargetScan Human (http://www.targetscan. org/vert_72/). The wild-type (WT) and mutated (MUT) KEL-FIB cells derived NR2F2 3'UTR vectors were constructed by Sangon Biotech Co., Ltd. Briefly, the full length WT NR2F2 3'UTR containing the binding site of miR-194-5p was amplified via PCR and cloned into the pmirGLO vector (Promega Corporation) between the Xhol and Notl sites to construct the pGLO-NR2F2-WT vector. DNA Polymerase was purchased from Invitrogen, Ltd. The thermocycling conditions were as follows: Initial denaturation at $94^{\circ} \mathrm{C}$ for $1 \mathrm{~min}$, 35 cycles of $94^{\circ} \mathrm{C}$ for $15 \mathrm{sec}$, annealing/elongation at $60^{\circ} \mathrm{C}$ for $30 \mathrm{sec}$ and a final extension at $72^{\circ} \mathrm{C}$ for $30 \mathrm{sec}$. As for mutated NR2F2 3'UTR, the binding site of NR2F2 3'UTR was mutated using a site-directed mutagenesis kit (Takara Bio, Inc.) to 5'-GGAUGAAUCAA-3' and amplified via PCR. The mutated NR2F2 3'UTR was inserted between the Xhol and Notl sites of the pmirGLO vector to construct the pGLO-NR2F2-MUT vector. KEL-FIB cells $\left(1 \times 10^{5}\right)$ were co-transfected with $0.8 \mu \mathrm{g}$ pGLO-NR2F2-WT or $0.8 \mu \mathrm{g}$ pGLO-NR2F2-MUT and $50 \mathrm{nM}$ miR-194-5p mimic or NC using Lipofectamine ${ }^{\circledR} 2000$ (Invitrogen; Thermo Fisher Scientific, Inc.). At $48 \mathrm{~h}$ post-transfection, luciferase activities were measured using the Dual-Luciferase Reporter Assay System (cat. no. E1910; Promega Corporation). Firefly luciferase activities were normalized to Renilla luciferase activities. Luciferase activity was averaged from three replicates. The primers used to perform mutagenesis are presented in Table SII.

Western blotting. Total protein was isolated from KEL-FIB cells using RIPA lysis buffer (Beijing Solarbio Science \& Technology Co., Ltd.), and the protein concentration was detected using BCA Protein Assay kit (Pierce; Thermo Fisher Scientific, Inc.). Total proteins (30 $\mu \mathrm{g} /$ lane) were separated via $10 \%$ SDS-PAGE and transferred to PVDF membranes, which were then blocked with $5 \%$ skimmed milk for $1 \mathrm{~h}$ at room temperature. Subsequently, the membranes were incubated at $4^{\circ} \mathrm{C}$ overnight with primary antibodies targeted against NR2F2 (1:1,000, cat. no. ab211777; Abcam) and GAPDH (1:1,000, cat. no. ab181602; Abcam). Following primary incubation, the membranes were incubated in Horseradish peroxidase (HRP)-conjugated secondary antibody (1:5,000, cat. no. ab205718; Abcam) for $2 \mathrm{~h}$ at room temperature. The membranes were washed three times with TBST containing $0.1 \%$ of Tween-20. Protein bands were visualized using ECL (EMD Millipore) and the ChemiDoc Touch Imaging system (Bio-Rad Laboratories, Inc.). Protein expression levels were semi-quantified using FluorChem FC2 system version 6.0.2 (Alpha Innotech Corporation) with GAPDH as the loading control.

Statistical analysis. Statistical analyses were performed using GraphPad Prism software (version 7.0; GraphPad Software, Inc.). Data are presented as the mean \pm SD from at least three independent experiments. Comparisons between two groups were analyzed using the unpaired Student's t-test. Comparisons among multiple groups were analyzed using one-way ANOVA followed by Dunnett's or Tukey's post hoc test. The correlation between NR2F2 mRNA expression and miR-194-5p expression was analyzed using Pearson's correlation analysis. $\mathrm{P}<0.05$ was considered to indicate a statistically significant difference.

\section{Results}

$N R 2 F 2$ is a key gene related to keloid pathology. The GSE7890 dataset was analyzed to identify key genes related to keloid pathology. A total of 104 DEGs with an adjusted P-value $<0.05$ were selected to perform GO and pathway enrichment analyses. The STRING analysis indicated that 'regulation of cell population proliferation', including 21 genes, was the most important biological process (Fig. 1A). The Metascape analysis of the 104 DEGs indicated that 'negative regulation of cell proliferation' and 'negative regulation of cell migration' were the key biological processes (Fig. 1B). The five overlapping genes (insulin like growth factor binding protein 5, pleiotrophin, T-box transcription factor 5, NR2F2 and DLC1 Rho GTPase activating protein) were selected from STRING and Metascape (Fig. 1C). Subsequently, we found that , NR2F2 was upregulated in keloid biopsies after literature review (23) so that NR2F2 was identified as the key gene closely associated with keloid pathology. The results also indicated that miR-194-5p might directly target NR2F2 (Fig. 1D).

miR-194-5p inhibits cell proliferation but promotes cell apoptosis in keloid fibroblasts. The RT-qPCR results indicated that miR-194-5p expression was decreased by 50\% in in KEL-FIB cells compared with HSF cells (Fig. 2A). Subsequently, KEL-FIB cells were transfected with miR-194-5p mimic and miR-194-5p inhibitor to further assess the function of miR-194-5p (Fig. 2B). The effects of miR-194-5p in KEL-FIB cells were investigated. The CCK- 8 assay results suggested that, compared with the NC group, miR-194-5p overexpression significantly inhibited cell proliferation, whereas miR-194-5p knockdown significantly increased KEL-FIB cell proliferation (Fig. 2C). Compared with the NC group (3.14 \pm 0.23$)$, the rate of apoptosis was increased by 3.44-fold in the miR-194-5p mimic group $(10.80 \pm 0.73)$, whereas the rate of apoptosis was decreased by $46 \%$ in the miR-194-5p inhibitor group

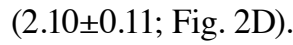

miR-194-5p inhibits keloid fibroblast migration and invasion. Following transfection with miR-194-5p mimic and miR-194-5p inhibitor, the effect of miR-194-5p on cell migration and invasion was assessed by performing wound healing and Transwell invasion assays, respectively. The results indicated that miR-194-5p overexpression decreased KEL-FIB cell migration by $14 \%$, whereas miR-194-5p knockdown increased KEL-FIB cell migration by 1.1 -fold compared with the NC group (Fig. 3A). Similarly, the Transwell invasion assay results indicated that miR-194-5p mimic decreased KEL-FIB cell invasion, whereas miR-194-5p inhibitor increased KEL-FIB cell invasion compared with the NC group (Fig. 3B).

Validation of NR2F2 as a direct target of miR-194-5p. The dual-luciferase reporter assay results indicated that miR-194-5p overexpression decreased the luciferase activity of NR2F2-WT by $50 \%$ compared with the NC group, whereas miR-194-5p overexpression did not significantly alter the luciferase activity of NR2F2-MUT (Fig. 4A). In addition, NR2F2 expression 
A

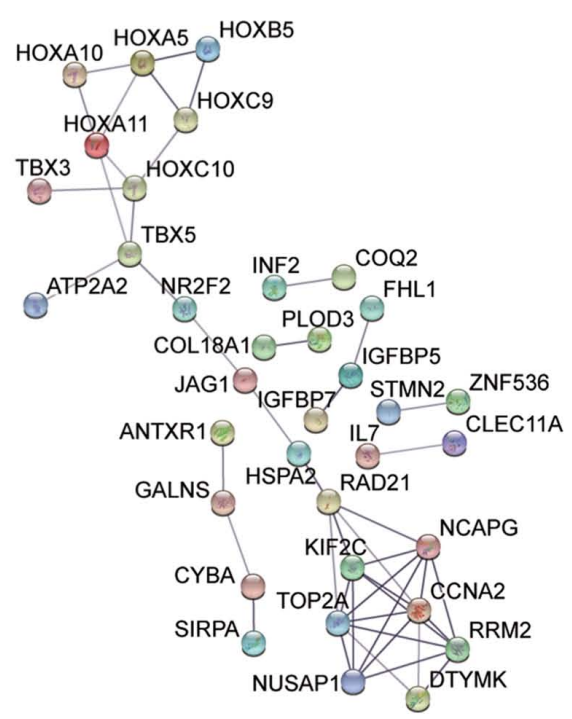

\begin{tabular}{|llll|}
\hline \multicolumn{4}{|c|}{ Biological process (GO) } \\
\hline GO-term & Description & $\begin{array}{c}\text { Count in } \\
\text { gene set }\end{array}$ & $\begin{array}{c}\text { False } \\
\text { discovery rate }\end{array}$ \\
\hline GO:0042127 & $\begin{array}{l}\text { Regulation of cell } \\
\text { population proliferation }\end{array}$ & 21 of 1594 & $8.46 \mathrm{E}-05$ \\
\hline GO:0009952 & $\begin{array}{l}\text { Anterior/posterior } \\
\text { pattern specification }\end{array}$ & 9 of 197 & $8.46 \mathrm{E}-05$ \\
\hline GO:0009887 & $\begin{array}{l}\text { Animal organ } \\
\text { morphogenesis }\end{array}$ & 16 of 865 & $8.46 \mathrm{E}-05$ \\
\hline GO:0045446 & $\begin{array}{l}\text { Endothelial } \\
\text { cell differentiation }\end{array}$ & 6 of 71 & $1.50 \mathrm{E}-04$ \\
\hline GO:0035295 & Tube development & 14 of 793 & $2.50 \mathrm{E}-04$ \\
\hline & & & (more...)
\end{tabular}

B
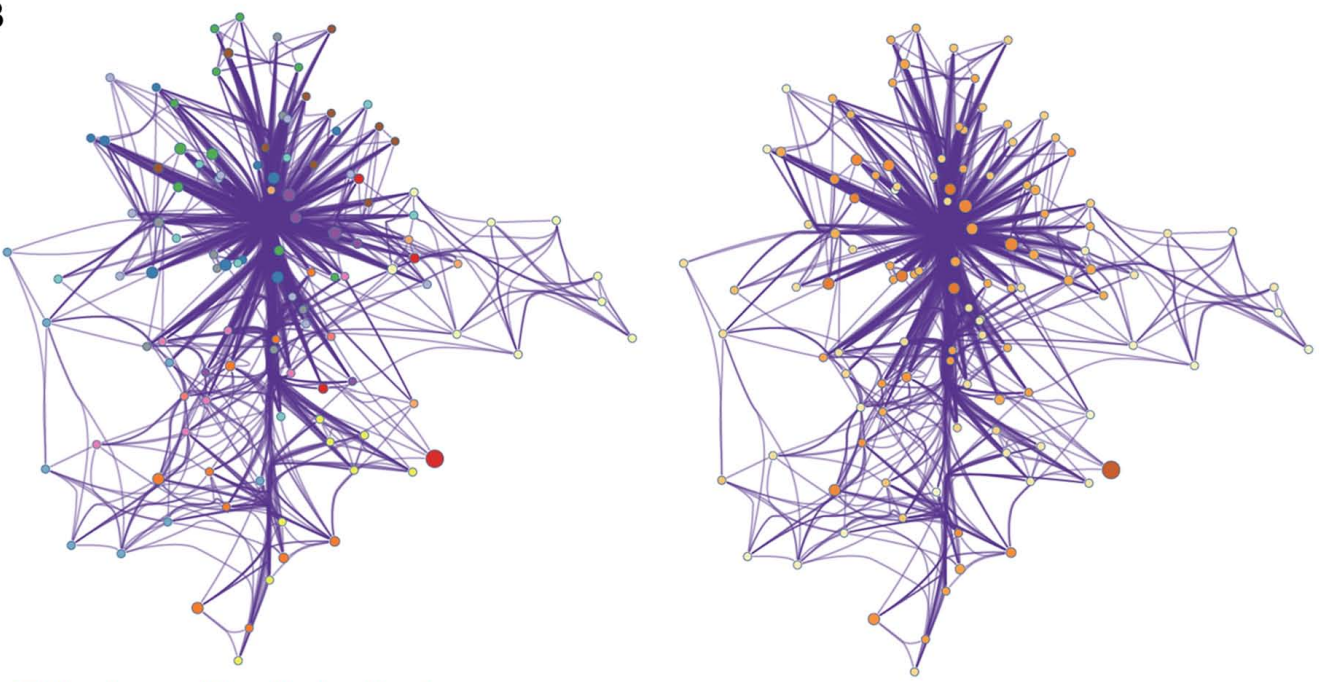

Negative regulation of cell proliferation

Negative regulation of cell migration

Cell fate commitment Blood vessel development

- Cellular response to organic cyclic compoud

Response to ketone Anterior/posterior pattern specification

Multi-multicellular organism process

- Gland morphogenesis प Tissue remodeling

Regulation of cell adhesion Epithelial cell development

Endocrine process Regulation of growth

Regulation of cell-substrate adhesion

C String

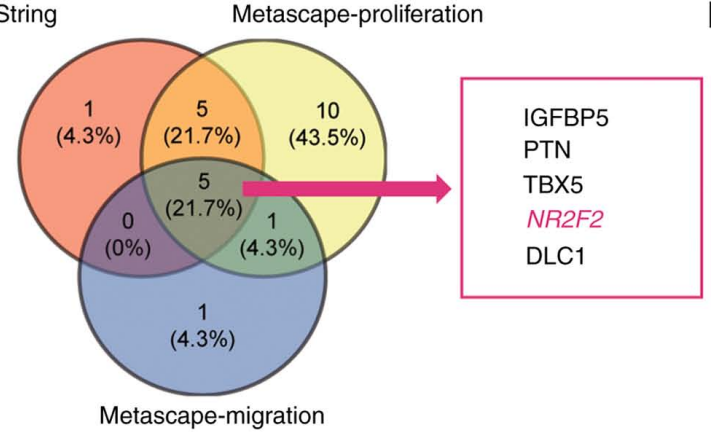

D

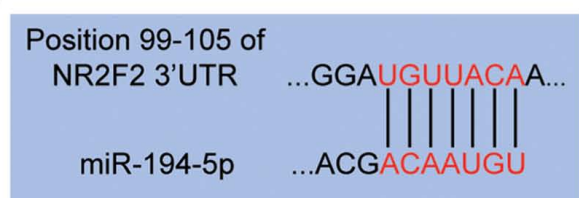

Figure 1. NR2F2 and miR-194-5p are the key mRNA and miRNA of interest in keloids, respectively. (A) The biological process analysis of 104 DEGs using STRING. A total of 104 DEGs were selected from the GSE7890 dataset, which was downloaded from Gene Expression Omnibus. STRING (string-db.org) is a database for functional enrichment analysis. (B) The GO and pathway analysis of 104 DEGs using Metascape. Metascape (metascape.org) is a visualized tool for the gene annotation and analysis. (C) The five overlapping genes (IGFBP5, PTN, TBX5, NR2F2 and DLC1) from STRING and Metascape analyses. (D) TargetScan Human was used to predict the binding site between NR2F2 mRNA 3'UTR and miR-194-5p. NR2F2, nuclear receptor subfamily 2 group F member 2; miR, microRNA; DEG, differentially expressed genes; STRING, Search Tool for the Retrieval of Interacting Genes/Proteins; GO, Gene Ontology; IGFBP5, insulin like growth factor binding protein 5; PTN, pleiotrophin; TBX5, T-box transcription factor 5; DLC1, DLC1 Rho GTPase activating protein; UTR, untranslated region. 

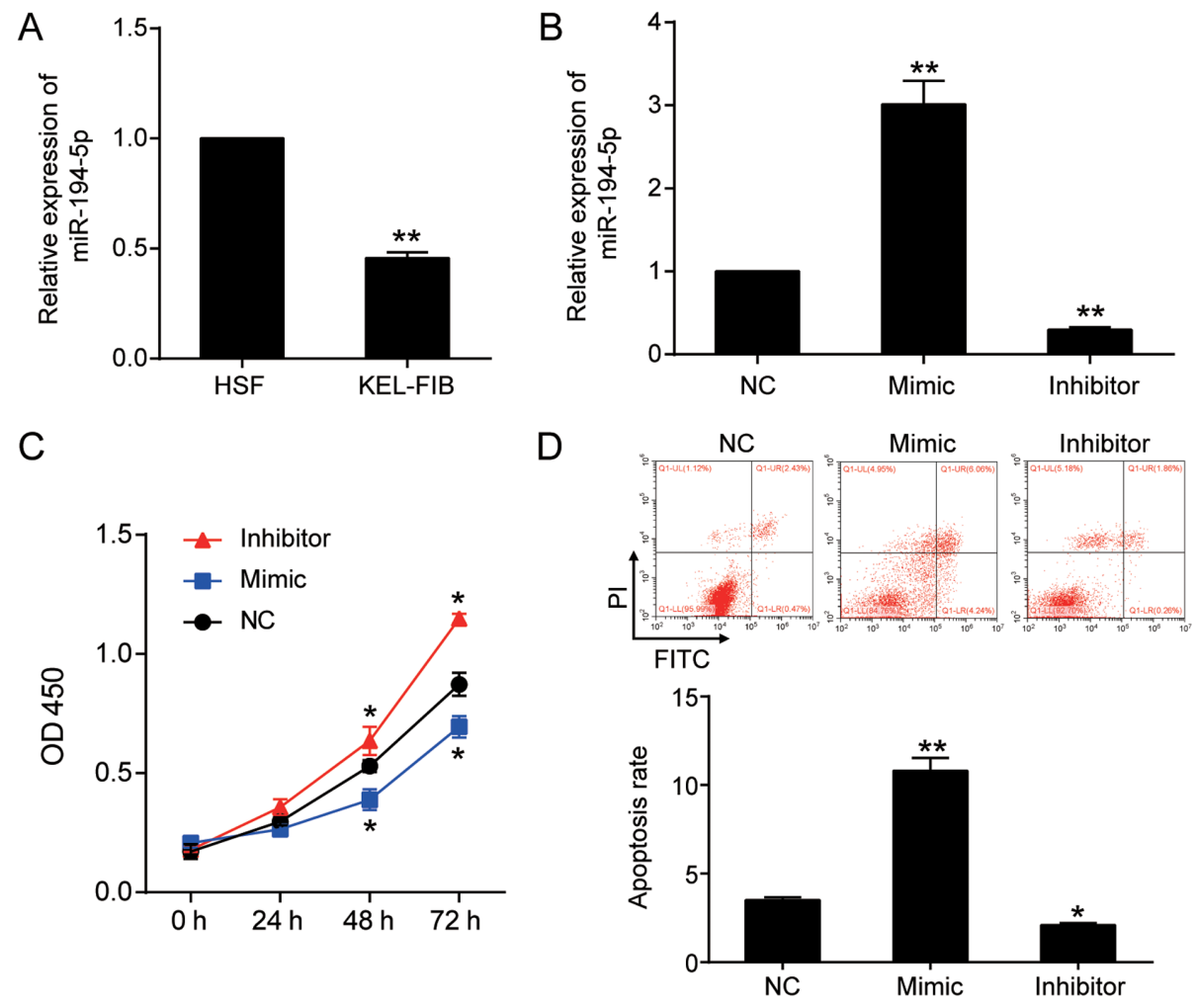

Figure 2. miR-194-5p expression is downregulated in keloids, and miR-194-5p inhibits cell proliferation but promotes cell apoptosis. (A) miR-194-5p expression levels in HSF and KEL-FIB cells. (B) miR-194-5p mimic and inhibitor were successfully transfected into KEL-FIB cells. (C) Cell proliferation was determined by performing the Cell Counting Kit- 8 assay at 24, 48 and $72 \mathrm{~h}$. (D) Flow cytometry was conducted to assess cell apoptosis. The apoptosis rate was calculated as the sum of late apoptosis (Q1-UR) and early apoptosis (Q1-LR). ${ }^{*} \mathrm{P}<0.05$ and ${ }^{* *} \mathrm{P}<0.001$ vs. HSF or NC. miR, microRNA; HSF, normal human skin fibroblasts cells; KEL-FIB, human keloid fibroblasts; NC, negative control; OD, optical density.

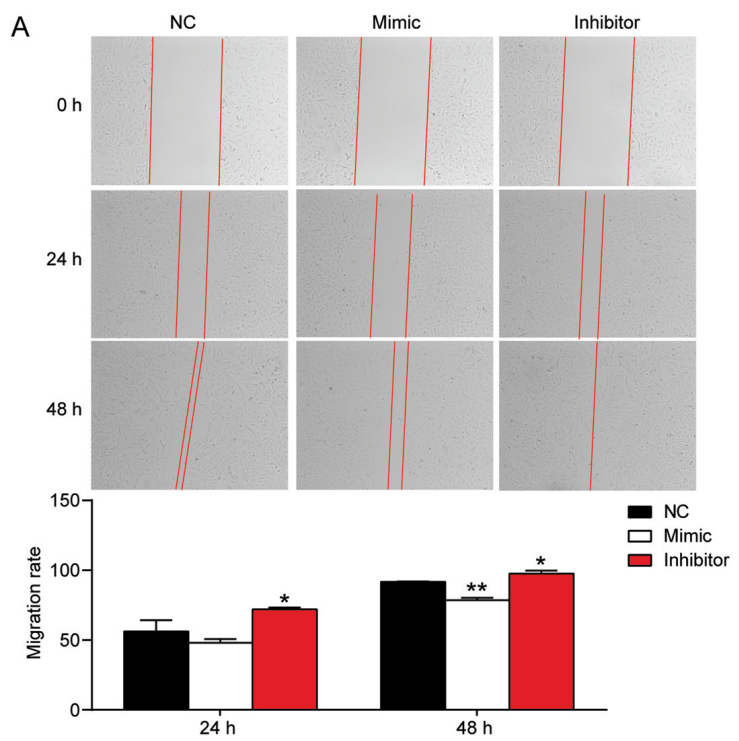

B
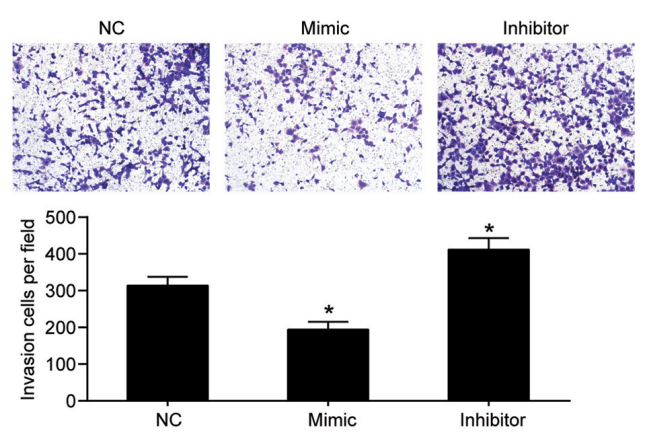

Figure 3. miR-194-5p inhibits cell migration and invasion. (A) The wound healing assay was performed to assess cell migration ability at 24 and $48 \mathrm{~h}$ (magnification, $\mathrm{x} 100$ ). The migration rate was calculated as the ratio of the wound healing distance at 24 or $48 \mathrm{~h}$ to the wound width at $0 \mathrm{~h}$. (B) The Transwell invasion assay was conducted to evaluate cell invasion (magnification, $\mathrm{x} 100$ ). ${ }^{*} \mathrm{P}<0.05$ and ${ }^{* * *} \mathrm{P}<0.001$ vs. NC. miR, microRNA; NC, negative control.

levels were increased by 3-fold in KEL-FIB cells compared with HSF cells (Fig. 4B). Pearson's correlation analysis indicated that NR2F2 and miR-194-5p expression levels were negatively correlated in KEL-FIB cells (Fig. 4C). Compared with the NC group, NR2F2 knockdown decreased NR2F2 expression, whereas miR-194-5p inhibitor increased NR2F2 expression at both the mRNA (Fig. 4D) and protein (Fig. 4E) levels.

$N R 2 F 2$ knockdown alters the aggressive phenotypes of keloid fibroblasts. To investigate the effect of NR2F2 on keloids in vitro, the CCK-8 assay was performed. The results indicated 
A

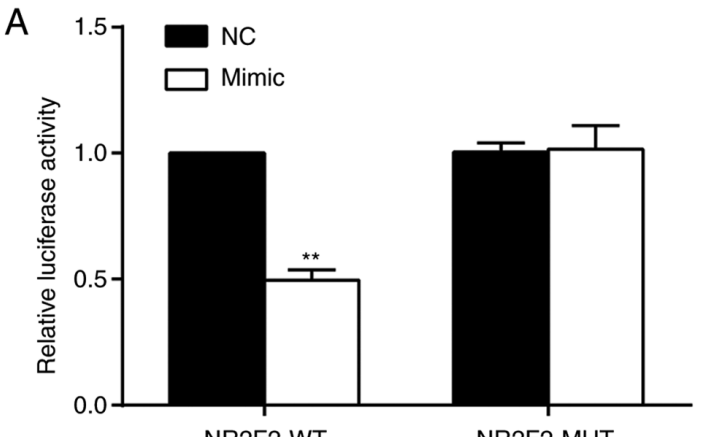

NR2F2-WT

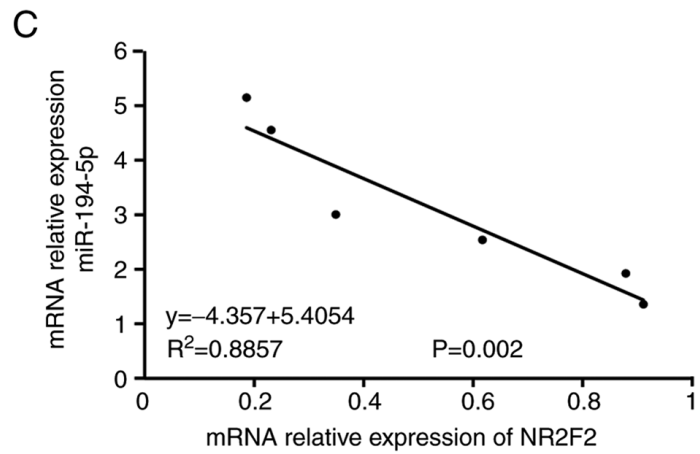

B

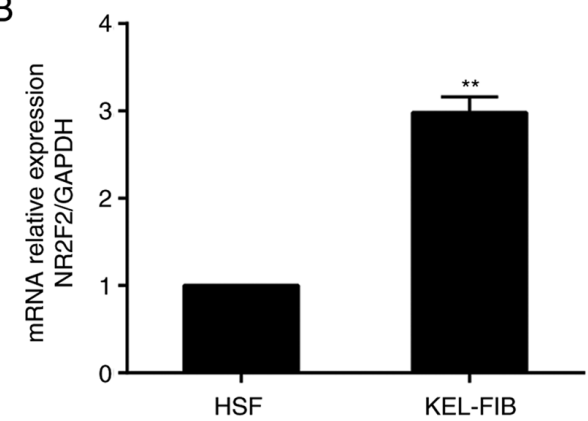

D

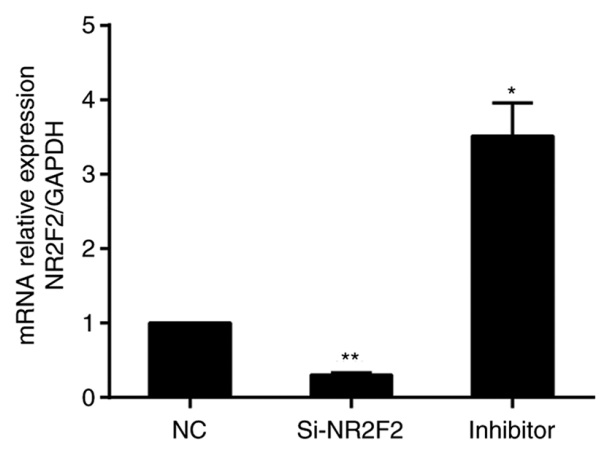

E

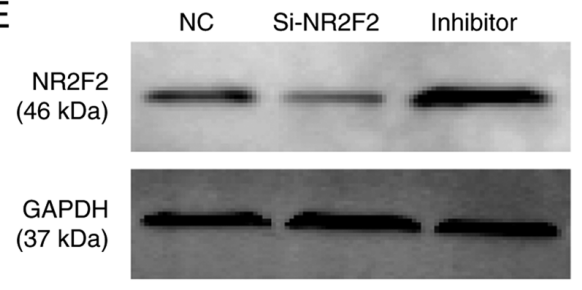

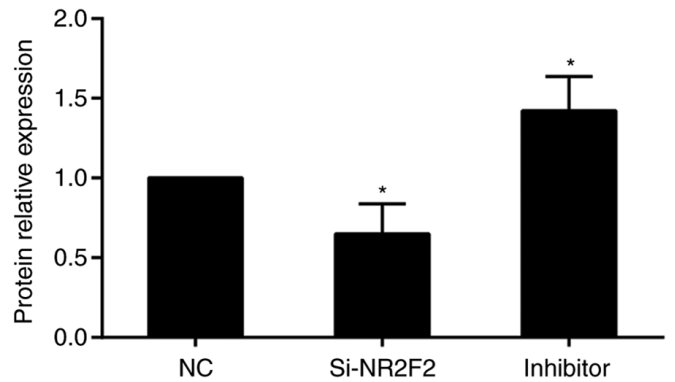

Figure 4. NR2F2 is a direct downstream target of miR-194-5p. (A) The dual-luciferase reporter assay results indicated that miR-194-5p mimic significantly inhibited luciferase activity in the NR2F2-WT group compared with NC mimic. (B) NR2F2 mRNA expression levels in HSF and KEL-FIB cells. (C) Pearson's correlation analysis indicated a negative correlation between the expression level of NR2F2 mRNA and miR-194-5p. NR2F2 (D) mRNA and (E) protein expression levels were measured via reverse transcription-quantitative PCR and western blotting, respectively. ${ }^{*} \mathrm{P}<0.05$ and ${ }^{* *} \mathrm{P}<0.001 \mathrm{vs}$. NC mimic or HSF. NR2F2, nuclear receptor subfamily 2 group F member 2; miR, microRNA; WT, wild-type; NC, negative control; HSF, normal human skin fibroblasts; KEL-FIB, human keloid fibroblasts; MUT, mutated; si, small interfering RNA.

that NR2F2 knockdown significantly decreased KEL-FIB cell proliferation at $72 \mathrm{~h}$ compared with the NC group (Fig. 5A). The rate of apoptosis was determined via flow cytometry, and the results suggested that the rate of apoptosis in the si-NR2F2 group was increased by 4.4-fold compared with the NC group (Fig. 5B). The migratory abilities of cells were evaluated by performing a wound healing assay. Compared with the $\mathrm{NC}$ group, cell migration in the si-NR2F2 group was decreased at $24(48 \pm 9.6$ vs. $71 \pm 1.7 \%)$ and $48 \mathrm{~h}(70 \pm 5.0$ vs. $87 \pm 3.5 \%)$ post-transfection (Fig. $5 \mathrm{C})$. The Transwell invasion assay indicated that si-NR2F2 decreased the number of invading cells compared with the NC group (Fig. 5D). Collectively, the results indicated that NR2F2 knockdown suppressed keloid progression in vitro.

NR2F2 knockdown reverses the influence of miR-194-5p inhibitor on keloid fibroblasts. To identify whether miR-194-5p regulated keloid fibroblasts by targeting NR2F2, a rescue experiment was performed. Co-transfection of miR-194-5p inhibitor and si-NR2F2 resulted in similar proliferation and invasion phenotypes to the $\mathrm{NC}$ group. The results suggested that NR2F2 knockdown reversed miR-194-5p inhibitor-mediated induction of KEL-FIB cell proliferation and invasion (Fig. 6A and B). Moreover, the CCK-8 assay results indicated that NR2F2 knockdown abolished the effects of miR-194-5p inhibitor at 48 and $72 \mathrm{~h}$. Similarly, the Transwell invasion assay results suggested that the number of invading cells in the co-transfection group was decreased by $38.7 \%(603 \pm 73)$ compared with the miR-194-5p inhibitor group (370 \pm 48 ; Fig. 6B). Therefore, the results indicated that miR-194-5p inhibited keloid progression by targeting NR2F2 in vitro.

\section{Discussion}

Keloids are a wound healing reaction due to abnormal skin injury, characterized by collagen deposition, as well as persistent fibrosis and inflammation $(25,26)$. The key pathogenic mechanism underlying keloids is not completely understood. In the present study, the results indicated that miR-194-5p expression 

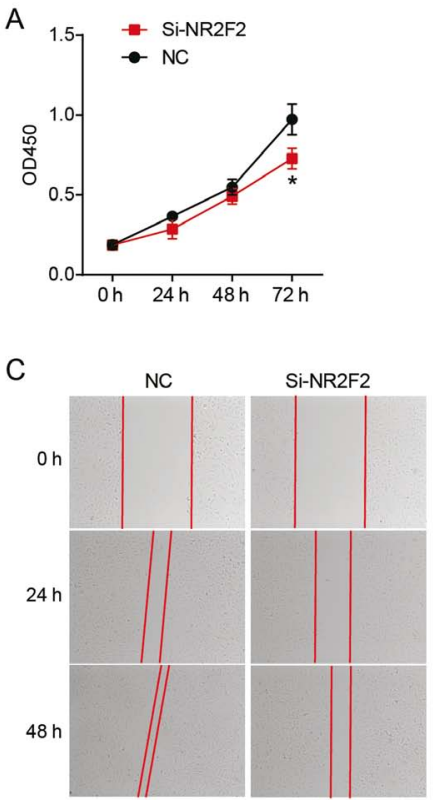

D

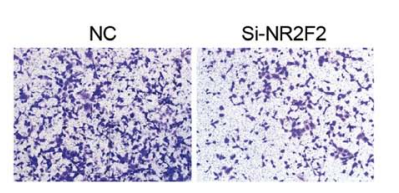

B
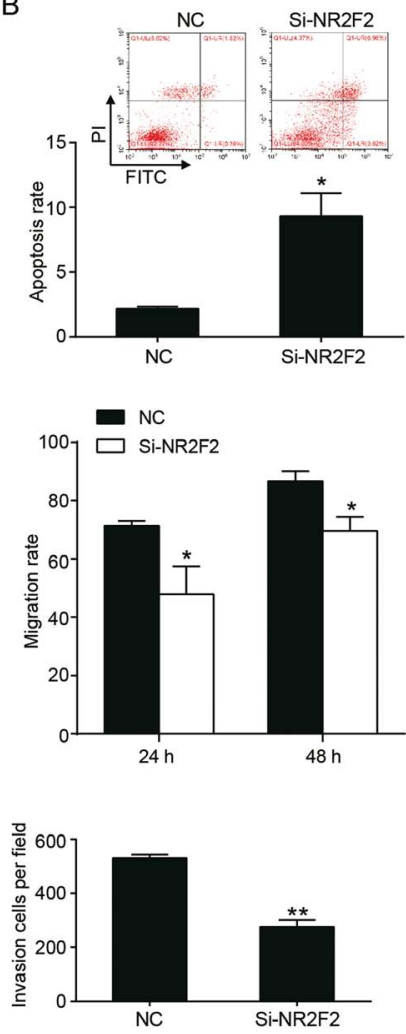

Figure 5. NR2F2 knockdown inhibits cell proliferation, invasion and migration, but promoted cell apoptosis in vitro. (A) Cell proliferation was assessed by performing a Cell Counting Kit- 8 assay. (B) Flow cytometry was conducted to assess cell apoptosis. The apoptosis rate was calculated as the sum of late apoptosis (Q1-UR) and early apoptosis (Q1-LR). (C) The wound healing assay was performed to assess cell migration at 24 and $48 \mathrm{~h}$ (magnification, $\mathrm{x} 100$ ). The migration rate was calculated as the ratio of the wound healing distance at 24 or $48 \mathrm{~h}$ to the wound width at $0 \mathrm{~h}$. (D) Cell invasion was determined by performing the Transwell invasion assay (magnification, $\mathrm{x} 100$ ). ${ }^{*} \mathrm{P}<0.05$ and ${ }^{* *} \mathrm{P}<0.001$ vs. NC. NR2F2, nuclear receptor subfamily 2 group F member 2 ; $\mathrm{NC}$, negative control; OD, optical density; si, small interfering RNA.

was significantly decreased in KEL-FIB cells compared with HSF cells. Compared with the NC group, miR-194-5p overexpression inhibited the aggressive phenotypes of keloid fibroblasts in vitro. Moreover, miR-194-5p was identified as a direct regulator of NR2F2 in KEL-FIB cells. Compared with the NC group, NR2F2 knockdown also inhibited the aggressive phenotypes of keloid fibroblasts, and promoted KEL-FIB cell apoptosis. Collectively, the results indicated that miR-194-5p suppressed keloid progression by targeting NR2F2.

miRNAs, consisting of 18-25 nucleotides, are highly conserved non-coding RNAs that can induce the degradation or translational inhibition of mRNA by binding to the 3'UTR of target genes (27,28). miRNAs have received increasing attention in medical research and have been reported to serve as tumor suppressor genes or proto-oncogenes (29). Previous studies have demonstrated that miRNAs are closely associated with the formation and development of keloids, which are benign tumors. In addition, Zhang et al (30) reported that miR-637 inhibited aggressive phenotypes by targeting Smad3 in human keloid fibroblast cells. In the present study, human keloid fibroblast cells were used to investigate the effects of miR-194-5p on the aggressive phenotypes of keloid fibroblast cells. The results indicated that miR-194-5p was downregulated KEL-FIB cells

A

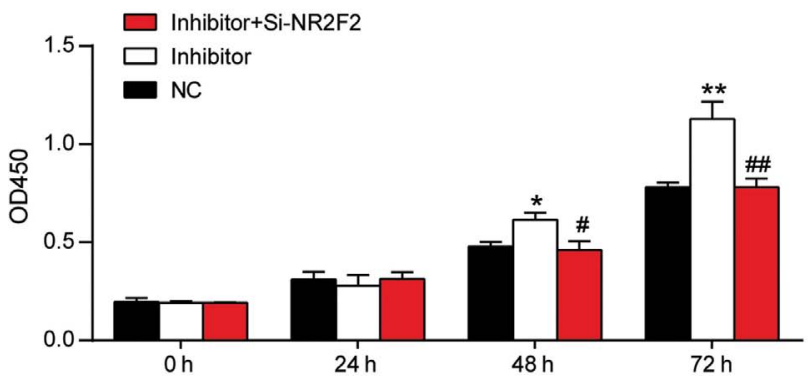

$\mathrm{B}$
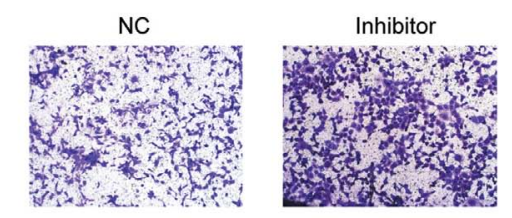

Inhibitor+Si-NR2F2

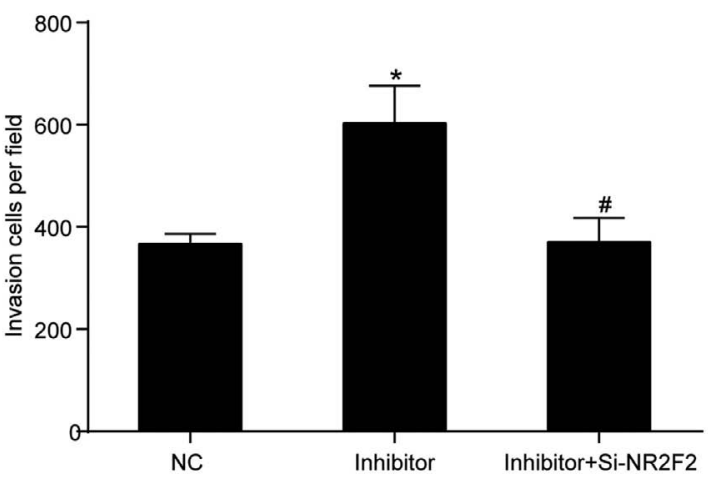

Figure 6. NR2F2 knockdown impaired miR-194-5p inhibitor-mediated effects on cell proliferation and invasion. (A) Cell proliferation was determined by performing the Cell Counting Kit- 8 assay. (B) Cell invasion was assessed by conducting a Transwell invasion assay (magnification, $\mathrm{x} 100) .{ }^{*} \mathrm{P}<0.05$ and ${ }^{* *} \mathrm{P}<0.001$ vs. $\mathrm{NC} ;{ }^{\#} \mathrm{P}<0.05$ and ${ }^{\# \#} \mathrm{P}<0.001$ vs. inhibitor. NR2F2, nuclear receptor subfamily 2 group F member 2; miR, microRNA; NC, negative control; si, small interfering RNA; OD, optical density.

compared with HSF cells, and miR-194-5p overexpression inhibited the aggressive phenotypes of keloid fibroblast cells compared with the NC group.

NR2F2, a member of the steroid/thyroid receptor superfamily, was screened out and predicted to be a miR-194-5p target gene via bioinformatics analysis in the present study. Previously, the biological significance of NR2F2 in prostate cancer was reported, demonstrating that NR2F2 was a therapeutic target and prognostic marker for prostate cancer via patient sample analysis (22). In vivo, NR2F2 knockdown suppressed pancreatic $\beta$ tumor cell invasion due to a defect in the angiogenic switch (31). Bao et al (32) demonstrated that NR2F2 overexpression was important for colorectal cancer metastasis, and promoted cell migration and metastasis in association with snail family transcriptional repressor 1 in vivo and in vitro. In line with the aforementioned previous studies, the present study investigated the hypothesis that NR2F2 might serve as a promoter in keloids. The results suggested that NR2F2 was upregulated in KEL-FIB cells compared with HSF cells, and NR2R2 knockdown effectively inhibited keloid fibroblast cell proliferation, migration and invasion, but promoted cell apoptosis compared with the NC group. In addition, the results suggested that NR2F2 3'UTR was regulated by miR-194-5p, and NR2F2 knockdown significantly reversed 
miR-194-5p inhibitor-mediated effects on the aggressive phenotypes of keloid fibroblasts.

However, the present study had a number of limitations that should be addressed in future studies. First, the KEL-FIB cell line was derived from a 35-year-old black female, whereas the HSF cell line was established in China, suggesting that the HSF cell line and KEL-FIB cell line might be derived from individuals with different ethnicities. A previous study reported that keloid formation in Caucasians was often accompanied by erythema and telangiectasia and pigmentation compared with keloid formation in African Americans (33); however, to the best of our knowledge, no previous study has investigated the difference in keloids between Asian and American individuals. In the present study, the HSF cell line (a normal skin fibroblast cell line) was used as a control cell line to assess the relative expression level of NR2F2 in the KEL-FIB cell line (a keloid fibroblast cell line). All functional experiments were performed using the KEL-FIB cell line to study the effects of NR2F2 on keloid fibroblasts. The HSF cell line was selected as the control cell line because, firstly, it has been widely used in previous keloid studies (34-36), and secondly, ATCC did not provide a normal skin fibroblast cell line. The lack of cell lines derived from individuals of the same ethnicity is a key limitation of the present study. Whether the miR-194-5p/NR2F2 interactome could serve as a diagnostic and therapeutic target for keloids also requires further investigation in vivo and validation using additional clinical characteristics data. Both in vivo assays and clinical-level correlation analyses should be conducted. In addition, the mechanism underlying the effect of NR2F2 in keloid fibroblasts should be investigated in future studies.

In summary, the present study indicated that miR-194-5p inhibited cell proliferation, migration and invasion, but promoted cell apoptosis in keloids. In addition, the results suggested that the expression of NR2F2 was downregulated by the upstream regulator miR-194-5p in keloid fibroblasts. Therefore, the present study indicated that the miR-194-5P/NR2F2 axis may serve as a potential biomarker or novel treatment strategy for keloids in the future.

\section{Acknowledgements}

Not applicable.

\section{Funding}

The present study was supported by the Wuhan University Young Teachers Funding Project (grant no. 2042018kf0145).

\section{Availability of data and materials}

The datasets used and or/analyzed during the current study are available from the corresponding author on reasonable request.

\section{Authors' contributions}

SJ designed the study and prepared the manuscript. QX supervised the study and interpreted the data. All authors read and approved the final manuscript.

\section{Ethics approval and consent to participate}

The present study was approved by the Ethics Committee of Renmin Hospital of Wuhan University (Wuhan, China).

\section{Patient consent for publication}

Not applicable.

\section{Competing interests}

The authors declare that they have no competing interests.

\section{References}

1. Patel PA, Bailey JK and Yakuboff KP: Treatment outcomes for keloid scar management in the pediatric burn population. Burns 38: 767-771, 2012.

2. Yao X, Cui X, Wu X, Xu P, Zhu W, Chen X and Zhao T: Tumor suppressive role of miR-1224-5p in keloid proliferation, apoptosis and invasion via the TGF- $\beta 1 / \mathrm{Smad} 3$ signaling pathway. Biochem Biophys Res Commun 495: 713-720, 2018.

3. Seifert $\mathrm{O}$ and Mrowietz U: Keloid scarring: Bench and bedside. Arch Dermatol Res 301: 259-272, 2009.

4. Finnerty CC, Jeschke MG, Branski LK, Barret JP, Dziewulski P and Herndon DN: Hypertrophic scarring: The greatest unmet challenge after burn injury. Lancet 388: 1427-1436, 2016.

5. Slemp AE and Kirschner RE: Keloids and scars: A review of keloids and scars, their pathogenesis, risk factors, and management. Curr Opin Pediatr 18: 396-402, 2006.

6. Berman B, Maderal A and Raphael B: Keloids and hypertrophic scars: Pathophysiology, classification, and treatment. Dermatol Surg 43 (Suppl 1): S3-S18, 2017.

7. Zhu W, Wu X, Yang B, YaoX, Cui X, Xu P and Chen X: miR-188-5p regulates proliferation and invasion via $\mathrm{PI} 3 \mathrm{~K} / \mathrm{Akt} / \mathrm{MMP}-2 / 9$ signaling in keloids. Acta Biochim Biophys Sin (Shanghai) 51: 185-196, 2019.

8. Yi R, O'Carroll D, Pasolli HA, Zhang Z, Dietrich FS, Tarakhovsky A and Fuchs E: Morphogenesis in skin is governed by discrete sets of differentially expressed microRNAs. Nat Genet 38: 356-362, 2006.

9. Chau BN and Brenner DA: What goes up must come down: The emerging role of microRNA in fibrosis. Hepatology 53: 4-6, 2011.

10. Li Z, Yu X, Shen J, Chan MT and Wu WK: MicroRNA in intervertebral disc degeneration. Cell Prolif 48: 278-283, 2015.

11. Yu X, Li Z and Liu J: MiRNAs in primary cutaneous lymphomas. Cell Prolif 48: 271-277, 2015

12. Li Z, Yu X, Shen J and Jiang Y: MicroRNA dysregulation in uveal melanoma: A new player enters the game. Oncotarget 6: 4562-4568, 2015.

13. Di Leva G, Garofalo M and Croce CM: MicroRNAs in cancer. Annu Rev Pathol 9: 287-314, 2014.

14. Ren L, Zhang J, Wang J, Wei J, Liu J, Li X, Zhu Y, Li Y, Guo C, Duan J, et al: Silica nanoparticles induce spermatocyte cell apoptosis through microRNA-2861 targeting death receptor pathway. Chemosphere 228: 709-720, 2019.

15. Zhao MJ, Xie J, Shu WJ, Wang HY, Bi J, Jiang W and Du HN: MiR-15b and miR-322 inhibit SETD3 expression to repress muscle cell differentiation. Cell Death Dis 10: 183, 2019.

16. Duan L, Duan D, Wei W, Sun Z, Xu H, Guo L and Wu X: MiR-19b-3p attenuates IL-1 $\beta$ induced extracellular matrix degradation and inflammatory injury in chondrocytes by targeting GRK6. Mol Cell Biochem 459: 205-214, 2019.

17. Li C, Bai Y, Liu H, Zuo X, Yao H, Xu Y and Cao M: Comparative study of microRNA profiling in keloid fibroblast and annotation of differential expressed microRNAs. Acta Biochim Biophys Sin (Shanghai) 45: 692-699, 2013.

18. Liu Y, Yang D, Xiao Z and Zhang M: miRNA expression profiles in keloid tissue and corresponding normal skin tissue. Aesthetic plastic surgery 36: 193-201, 2012.

19. Navab R, Gonzalez-Santos JM, Johnston MR, Liu J, Brodt P, Tsao MS and $\mathrm{Hu} \mathrm{J}$ : Expression of chicken ovalbumin upstream promoter-transcription factor II enhances invasiveness of human lung carcinoma cells. Cancer Res 64: 5097-5105, 2004. 
20. Litchfield LM, Appana SN, Datta S and Klinge CM: COUP-TFI inhibits NFkappaB activation in endocrine-resistant breast cancer cells. Mol Cell Endocrinol 382: 358-367, 2014.

21. Zhang W, Liu J, Qiu J, Fu X, Tang Q, Yang F, Zhao Z and Wang H: MicroRNA-382 inhibits prostate cancer cell proliferation and metastasis through targeting COUP-TFII. Oncol Rep 36: 3707-3715, 2016.

22. Qin J, Wu SP, Creighton CJ, Dai F, Xie X, Cheng CM, Frolov A, Ayala G, Lin X, Feng XH, et al: COUP-TFII inhibits TGF- $\beta$-induced growth barrier to promote prostate tumorigenesis. Nature 493: 236-240, 2013.

23. Smith JC, Boone BE, Opalenik SR, Williams SM and Russell SB: Gene profiling of keloid fibroblasts shows altered expression in multiple fibrosis-associated pathways. J Invest Dermatol 128 : 1298-1310, 2008.

24. Livak KJ and Schmittgen TD: Analysis of relative gene expression data using real-time quantitative PCR and the 2(-Delta Delta C(T)) method. Methods 25: 402-408, 2001.

25. Sidgwick GP and Bayat A: Extracellular matrix molecules implicated in hypertrophic and keloid scarring. J Eur Acad Dermatol Venereol 26: 141-152, 2012.

26. Huang $C$ and Ogawa R: The link between hypertension and pathological scarring: Does hypertension cause or promote keloid and hypertrophic scar pathogenesis? Wound Repair Regen 22 462-466, 2014.

27. Rutnam ZJ, Wight TN and Yang BB: miRNAs regulate expression and function of extracellular matrix molecules. Matrix Biol 32: 74-85, 2013.

28. Meltzer PS: Cancer genomics: Small RNAs with big impacts. Nature 435: 745-746, 2005.
29. Price $\mathrm{C}$ and Chen J: MicroRNAs in cancer biology and therapy: Current status and perspectives. Genes Dis 1: 53-63, 2014.

30. Zhang Y, Guo B,Hui Q, Li W, Chang Pand Tao K: Downregulation of miR-637 promotes proliferation and metastasis by targeting Smad3 in keloids. Mol Med Rep 18: 1628-1636, 2018.

31. Qin J, Chen X, Yu-Lee LY, Tsai MJ and Tsai SY: Nuclear receptor COUP-TFII controls pancreatic islet tumor angiogenesis by regulating vascular endothelial growth factor/vascular endothelial growth factor receptor-2 signaling. Cancer Res 70 : 8812-8821, 2010.

32. Bao Y, Gu D, Feng W, Sun X, Wang X, Zhang X, Shi Q, Cui G, $\mathrm{Yu} \mathrm{H}$, Tang $\mathrm{C}$ and Deng A: COUP-TFII regulates metastasis of colorectal adenocarcinoma cells by modulating Snail1. Br J Cancer 111: 933-943, 2014.

33. Naylor MC and Brissett AE: Current concepts in the etiology and treatment of keloids. Facial Plast Surg 28: 504-512, 2012.

34. Hou Z, Fan F and Liu P: BTXA regulates the epithelial-mesenchymal transition and autophagy of keloid fibroblasts via modulating miR-1587/miR-2392 targeted ZEB2. Biosci Rep 39: BSR20190679, 2019.

35. Jin J, Zhai HF, Jia ZH and Luo XH: Long non-coding RNA HOXA11-AS induces type I collagen synthesis to stimulate keloid formation via sponging miR-124-3p and activation of Smad5 signaling. Am J Physiol Cell Physiol 317: C1001-C1010, 2019.

36. Jin J, Jia ZH, Luo XH and Zhai HF: Long non-coding RNA HOXA11-AS accelerates the progression of keloid formation via miR-124-3p/TGFßR1 axis. Cell Cycle 19: 218-232, 2020. 